\title{
Phenotypic features of chronic migraine
}

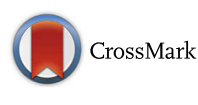

\author{
Osman Özgür Yalın*, Derya Uluduz², Aynur Özge ${ }^{3}$, Mehmet Ali Sungur ${ }^{4}$, Macit Selekler $^{5}$ and Aksel Siva ${ }^{2}$
}

\begin{abstract}
Background: Chronic migraine is a disabling, under-recognized, and undertreated disorder that increases health burdens. The aim of this study was to evaluate phenotypic features and the relevance of accompanying symptoms of migraine attacks in chronic migraine.

Method: This study was conducted as part of an ongoing Turkish Headache Database Study investigating the clinical characteristics and outcomes of headache syndromes in the Turkish population. The electronic database was examined retrospectively, and 835 patients with chronic migraine were included.

Results: Patient group consisted of 710 women and 125 men (85 and $15 \%$, respectively). Mean patient age was 36.8 \pm 13.5 years, median value of migraine onset was 60 months (18-120), median headache frequency was 25 days per month (16-30), median of attack duration was $12 \mathrm{~h}$ (4-24), and median of intensity was eight (7-9). Increasing headache days per month were inversely related with the presence of nausea, vomiting, phonophobia, and photophobia. Longer duration of headache (months) and higher visual analog scale (VAS) for headache intensity were associated with all accompanying symptoms. Phonophobia, nausea, photophobia, and vomiting were the most frequent accompanying symptoms (experienced by $80.2,77.6,71.2$, and $40.9 \%$ of patients, respectively). Osmophobia was also frequent in chronic migraine patients (53.4\%) and was closely associated with other accompanying symptoms. Vertigo and dizziness were observed less frequently, and they were not associated with accompanying symptoms.

Conclusion: Phenotype of chronic migraine may be associated with the course of chronification. Duration of illness and attack intensity were closely related with the presence of accompanying symptoms, although headache frequency was found to be inversely related to the presence of accompanying symptoms. Osmophobia was also a frequent symptom and was closely related with other accompanied symptoms, unlike vertigo and dizziness. Inclusion of osmophobia into the diagnostic criteria might improve accurate diagnosis of chronic migraine.
\end{abstract}

Keywords: Chronic migraine, Phenotype, Accompanying symptoms, Chronification

\section{Background}

Chronic migraine (CM) is defined by the presence of headache attacks on 15 days or more per month for at least three months of which 8 or more days meet criteria for migraine [1]. A prevalence rate of $2 \%$ for $\mathrm{CM}$ has been estimated [2-4]. Although CM is much less common than episodic migraine (EM), it is substantially more debilitating, with a greater negative effect on quality of life, and it is a challenging disorder for practitioners $[4,5]$. Despite the substantial personal and social

\footnotetext{
* Correspondence: osmanozguryalin@yahoo.com

${ }^{1}$ Neurology Department, Istanbul Education and Research Hospital, Kasap Illyas Mah. Org. Abdurrahman, Nafiz Gürman Cd, PK: 34098 Fatih, Istanbul, Turkey

Full list of author information is available at the end of the article
}

consequences of CM, its diagnostic criteria are still evolving [6]. The third edition of the International Classification of Headache Disorders (ICHD-3 beta) [1], published in 2013, has comprehensively described primary chronic headache disorders, such as $\mathrm{CM}$, chronic tension-type headache, new daily persistent headache, and chronic hemicrania continua [1]. In this new edition, medication overuse headache is not an exclusion criterion; therefore, CM now represents a broader patient group.

$\mathrm{CM}$ is a disabling, underdiagnosed, and undertreated disorder [6]. CM patients constitute the most debilitated subgroup of the migraine population, and may therefore represent a clinically distinct group [7]. According to the American Migraine Prevalence and Prevention study, patients with $\mathrm{CM}$ were twice as likely to develop depression,
Springer

(C) 2016 Yalın et al. Open Access This article is distributed under the terms of the Creative Commons Attribution 4.0 International License (http://creativecommons.org/licenses/by/4.0/), which permits unrestricted use, distribution, and reproduction in any medium, provided you give appropriate credit to the original author(s) and the source, provide a link to the Creative Commons license, and indicate if changes were made. 
anxiety, and chronic pain than those with EM [8]. Cutaneous allodynia, anxiety, disability, and depression are frequently considered as predictors of chronification $[9,10]$.

The explicit mechanisms of CM are not fully understood. Atypical modulation of pain may play a role in its pathogenesis [11]. Pain modulatory pathway alterations have been identified in CM [12]. A suggested mechanism for chronification of migraine is that repeated migraine attacks could have cumulative structural effects on the trigeminovascular pathway [13]. This theory is supported by the evidence of iron deposition in the periaqueductal gray matter of patients with a long history of migraine [14]. In addition, CM is associated with a greater degree of impairment in cortical processing of sensory stimuli [15]. Therefore, proposed structural changes in the excitability of the trigeminovascular pathway and/or other brainstem pain modulating systems could result in different phenotypes of the disease.

According to the ICHD-3 beta diagnostic criteria of migraine, accompanying symptoms item (D) require either (1) nausea and/or vomiting and/or (2) photophobia and phonophobia. Although osmophobia is mentioned as an accompanying symptom in the appendix-definitions section, it has not been included in the diagnostic criteria. Osmophobia refers to an increased sensitivity to odors during migraine attacks, and it seems to be a distinguishing feature of migraine [16]. Association between osmophobia and allodynia has also been investigated recently, and it is also proposed as a shared central sensitization mechanism [17].

To the best of our knowledge, the association between headache characteristics and accompanying symptoms of CM has not been reported yet. The aims of this study are (1) to determine the clinical characteristics of headache attacks and to investigate the relevance of associated symptoms such as nausea, vomiting, phonophobia, and photophobia; (2) to investigate other associated symptoms that could improve diagnostic certainty, including osmophobia, dizziness, and vertigo; and (3) to analyze relationships among associated symptoms.

\section{Methods}

This retrospective observational study was a part of ongoing Turkish Headache Database (THD) Study. THD investigating the clinical characteristics and outcomes of primary and secondary headache syndromes in Turkish population is supported by Turkish Neurological Society. Out of tertiary headache centers in THD, patients diagnosed with CM based on ICHD-2 criteria between the years 2008 and 2012 in Mersin University, Istanbul University and Kocaeli University were enrolled to this study. The Human Ethical Committee of Mersin University (MEU. 0.01.00.06/265, 20.10.2008) approved this nonprofit study. Subjects meeting the inclusion criteria were enrolled into the study after giving written consent. Diagnosis of migraine was done by headache experts with faceto-face examinations according to ICHD-2 criteria [18]. Subjects younger than 18 years of age and those older than 80 years of age ( $n=123$ subjects), those who had received a headache diagnosis other than CM $(n=2850$ episodic migraine subjects), and patients with chronic $\mathrm{TTH}$, hemicrania continua, medication overuse headache and patients who had TTH attacks more frequently than migraine attacks ( $n=385$ subjects) were excluded. Data on socio-demographic and clinical characteristics, including age at onset, headache duration, headache frequency, presence of aura, headache characteristics, localization, intensity of pain, triggering factors, associated features, comorbidity, and medical and family history were gathered from a web-based database. Accompanying symptoms of CM were evaluated based on ICHD-2 criteria. Presence of vertigo, dizziness and osmophobia were also analyzed retrospectively taking into consideration of ICHD-3 beta criteria. Vertigo and dizziness is stated when related with migraine attacks. Vertigo definition based to clinical features: internal/external vertigo, positional, occurring during head motion. Similarly dizziness defined sensation of disequilibrium occurring during headache attack, discrimination is particularly based to clinical examination of experts.

When extracting data from the electronic database, we excluded all missing data, including missing data on accompanying symptoms. Therefore, we obtained a reduced number of subjects in osmophobia assessments. Although this could limit the power of the analysis, it also increased the reliability of the data and prevented errors that could be attributed to the retrospective design of the study. The visual analog scale (VAS) was used for assessment of pain intensity.

\section{Statistical analysis}

Data were entered and analyzed in the statistical analysis program PASW v.18 (SPSS Inc. Released 2009, PASW Statistics for Windows, Version 18.0. Chicago: SPSS Inc.) statistical package. Distributions of data were examined with the Shapiro-Wilk test, and independent samples $t$ test and one-way ANOVA or non-parametric alternatives (Mann-Whitney U and Kruskal-Wallis tests) were used to compare groups according to the distribution of dependent variables. Continuous variables were summarized as mean \pm standard deviation or median (25-75\%) according to the distribution, and categorical variables were summarized as frequency and percentile. Spearman nonparametric correlations were used to analyze relationships among headache properties. Pearson-Chi square or Fisher's exact test were used according to the expected count rule for categorical data. Two-tailed $p<0.05$ was accepted as significant. 


\section{Results}

The study group consisted of $835 \mathrm{CM}$ patients, 710 female $(85 \%)$, mean age $36.8 \pm 13.5$ years. The median value of migraine onset was 60 months (18-120), median headache duration was $12 \mathrm{~h}(4-24)$ and median headache intensity was eight (7-9). Seventy-seven percent of CM patients described throbbing headache followed by pressing (13.7 \%) and dull (5.5 \%) headache. The localization of headache was predominantly unilateral (39.5\%), followed by bifrontal (10.7\%), generalized (14.8\%), and sub-occipital (13.6\%). Phonophobia (80.2 \%) was the most common accompanying symptom, followed by nausea $(77.6 \%)$ and photophobia (71.2 \%). Vomiting was present in $40.9 \%$ of patients. Osmophobia was observed in $53.4 \%$ of patients, and dizziness and vertigo was present in $17.5 \%$ and $14.4 \%$ of patients respectively. Characteristics of study patients is summarized at Table 1 . The most common triggering factor was emotional stress (79.2 \%), followed by skipping meals (57.0\%), menstruation (36.7 \%), and seasonal factors $(23.0 \%)$. Most of the patients reported no intradaydifference, but for $31.4 \%$ of patients, headaches were most intense in the evening. Headache frequency did not change with age. Headache frequency was inversely

Table 1 Headache characteristics of study group

\begin{tabular}{|c|c|c|}
\hline \multirow{2}{*}{ Gender } & Female & $710(85 \%)$ \\
\hline & Male & $125(15 \%)$ \\
\hline \multirow[t]{2}{*}{ Age } & Age & $36,8 \pm 13,5$ \\
\hline & Duration of illness (months) & $60(18-120)$ \\
\hline \multirow{4}{*}{ Headache characteristics } & Headache frequency (days/month) & $25(16-30)$ \\
\hline & Headache duration (hours) & $12(4-24)$ \\
\hline & Headache intensity (VAS) & $8(7-9)$ \\
\hline & Throbbing headache & $631(77.2 \%)$ \\
\hline \multirow{5}{*}{$\begin{array}{l}\text { Quality of headache } \\
(n=817)\end{array}$} & Pressing & $112(13.7 \%)$ \\
\hline & Dull & $45(5.5 \%)$ \\
\hline & Others & $29(3.5 \%)$ \\
\hline & Unilateral & $320(39.5 \%)$ \\
\hline & Bifrontal & $87(10.7 \%)$ \\
\hline \multirow{6}{*}{$\begin{array}{l}\text { Headache localization } \\
(n=811)\end{array}$} & Generalized & $120(14.8 \%)$ \\
\hline & Sub-occipital & $110(13.6 \%)$ \\
\hline & Others & $198(21.4 \%)$ \\
\hline & Phonophobia $(n=792)$ & $635(80.2 \%)$ \\
\hline & Nausea $(n=789)$ & $612(77.6 \%)$ \\
\hline & Photophobia $(n=787)$ & $560(71.2 \%)$ \\
\hline \multirow{4}{*}{$\begin{array}{l}\text { Accompanying } \\
\text { symptoms }\end{array}$} & Vomiting $(n=738)$ & $302(40.9 \%)$ \\
\hline & Osmophobia $(n=238)$ & $127(53.4 \%)$ \\
\hline & Dizziness $(n=661)$ & $116(17.5 \%)$ \\
\hline & Vertigo $(n=641)$ & $92(14.4 \%)$ \\
\hline
\end{tabular}

VAS visual analog scale related with headache onset $(r=-0.131, p<0.001)$, attack duration $(r=-0.110, p=0.002)$, and intensity of headache $(r=-0.118, p=0.002)$. When correlation analysis adjusted according to gender and age covariates; correlation of headache onset disappeared, headache frequency was inversely related with attack duration $(r=-0.083, p=0.032)$, and intensity of headache $(r=-0.139, p<0.001)$. Analysis of gender effects revealed no difference between males and females in terms of headache frequency $(p=0.589)$, duration of illness $(p=0.054)$, or intensity $(p=0.559)$. Attack duration (hours) was significantly longer for females $(p<0.001)$ (Table 2).

To reveal the phenotypic features of $\mathrm{CM}$, we compared headache frequency, duration of attack, intensity, and duration of illness according to the presence of accompanying symptoms. First, we compared headache frequency (headache days per month) in terms of the presence of accompanying symptoms. We observed that the presence of nausea, vomiting, phonophobia, and photophobia were significantly associated with fewer headache days per month (Table 3). When we investigated attack duration, we observed that vomiting, phonophobia, and photophobia were associated with longer duration of attack (Table 4). Greater headache intensity (VAS) and longer duration of illness (in months) were significantly associated with the existence of all accompanying symptoms, including nausea, vomiting, phonophobia, photophobia, dizziness, vertigo, and osmophobia (see Tables 5 and 6 for intensity and duration, respectively).

We also analyzed the associations of accompanying symptoms with each other (Table 7). The diagnostic accompanying symptoms described in ICHD-2 and also in ICHD-3 beta are nausea, vomiting, phonophobia, and photophobia, and these symptoms were found to be closely related with each other $(p=0.001)$. The crosstab analysis revealed that osmophobia and dizziness were significantly correlated with the presence of other diagnostic accompanying symptoms. By using $x^{2}$ tests, we found that osmophobia was associated with more frequent incidences of nausea $(p=0.006)$, vomiting $(p=0.013)$, phonophobia $(p=0.001)$, and photophobia $(p=0.001)$. Dizziness was associated with more frequent incidences of nausea $(p=0.008)$, vomiting $(0.001)$, phonophobia $(p=0.001)$, and photophobia $(p=0.001)$. The presence of vertigo was

Table 2 Headache characteristics according to gender

\begin{tabular}{llll}
\hline & $\begin{array}{l}\text { Male } \\
(n=125)\end{array}$ & $\begin{array}{l}\text { Female } \\
(n=710)\end{array}$ & $p$ \\
\hline Headache frequency (days/month) & $20[16-30]$ & $25[16-30]$ & 0.589 \\
Duration of illness (months) & $48[12-120]$ & $60[24-132]$ & 0.054 \\
Headache duration (hours) & $7[2-24]$ & $12[4-24]$ & $<0.001$ \\
Headache intensity (VAS) & $8[7-9]$ & $8[7-9]$ & 0.559 \\
\hline
\end{tabular}

VAS visual analog scale 
Table 3 Headache frequency (days/month) according to accompanying symptoms

\begin{tabular}{lllll}
\hline $\begin{array}{l}\text { Accompanying } \\
\text { symptoms }\end{array}$ & & $\begin{array}{l}\text { Number of } \\
\text { subjects }(n)\end{array}$ & $\begin{array}{l}\text { Headache days/ } \\
\text { month }\end{array}$ & $P$ value \\
\hline Nausea & Present & 612 & $20[15-30]$ & 0.008 \\
& Absent & 177 & $30[16-30]$ & \\
Vomiting & Present & 302 & $20[15-30]$ & $<0.001$ \\
& Absent & 436 & $30[18-30]$ & \\
Phonophobia & Present & 635 & $20[15-30]$ & 0.002 \\
& Absent & 157 & $30[20-30]$ & \\
Photophobia & Present & 560 & $20[15-30]$ & $<0.001$ \\
& Absent & 227 & $30[20-30]$ & \\
Dizziness & Present & 116 & $25[16-30]$ & 0.056 \\
& Absent & 545 & $20[16-30]$ & \\
Vertigo & Present & 92 & $25[16-30]$ & 0.440 \\
& Absent & 549 & $24[16-30]$ & \\
Osmophobia & Present & 127 & $20[16-30]$ & 0.371 \\
& Absent & 111 & $20[15-30]$ & \\
\hline
\end{tabular}

not associated with nausea $(p=0.445)$, vomiting $(p=0.142)$, or photophobia $(p=0.063)$. A detailed analysis of the accompanying symptoms is presented in Table 7.

\section{Discussion}

Our study revealed that the presence of diagnostic accompanying symptoms (nausea, vomiting, phonophobia, and photophobia) lessened with higher numbers of headache days per month. This finding is compatible with the literature. Migraine characteristics, especially accompanying symptoms, are less prominent in the CM population [19].

Table 4 Headache duration (hours) according to accompanying symptoms

\begin{tabular}{lllll}
\hline $\begin{array}{l}\text { Accompanying } \\
\text { symptoms }\end{array}$ & & $\begin{array}{l}\text { Number of } \\
\text { subjects }(n)\end{array}$ & $\begin{array}{l}\text { Headache duration } \\
\text { (hours) }\end{array}$ & P value \\
\hline Nausea & Present & 612 & $12[4-24]$ & 0.056 \\
Vomiting & Absent & 177 & $12[3-24]$ & \\
& Present & 302 & $19[6-24]$ & $<0.001$ \\
Phonophobia & Absent & 436 & $12[3-24]$ & \\
& Present & 635 & $12[4-24]$ & 0.010 \\
Photophobia & Absent & 157 & $11[4-24]$ & \\
& Present & 560 & $12[4-24]$ & 0.003 \\
Dizziness & Absent & 227 & $10[3-24]$ & \\
& Present & 116 & $12[6-24]$ & 0.148 \\
Vertigo & Absent & 545 & $12[4-24]$ & \\
& Present & 92 & $12[5-24]$ & 0.826 \\
Osmophobia & Present & 127 & $12[7-24]$ & 0.219 \\
& Absent & 111 & $12[4-24]$ & \\
\hline
\end{tabular}

Table 5 Headache intensity (VAS) according to accompanying symptoms

\begin{tabular}{lllll}
\hline $\begin{array}{l}\text { Accompanying } \\
\text { symptoms }\end{array}$ & & $\begin{array}{l}\text { Number of } \\
\text { subjects ( } n \text { ) }\end{array}$ & $\begin{array}{l}\text { Headache intensity } \\
\text { (VAS) }\end{array}$ & $P$ value \\
\hline Nausea & Present & 612 & $8[7-10]$ & $<0.001$ \\
Vomiting & Absent & 177 & $7[6-9]$ & \\
& Present & 302 & $8[7-10]$ & $<0.001$ \\
Phonophobia & Absent & 436 & $7[6-9]$ & \\
& Present & 635 & $8[7-10]$ & $<0.010$ \\
Photophobia & Absent & 157 & $7[6-8]$ & \\
& Present & 560 & $8[7-10]$ & $<0.001$ \\
Dizziness & Absent & 227 & $7[6-8]$ & 0.004 \\
& Present & 116 & $8[7-10]$ & \\
Vertigo & Absent & 545 & $8[7-9]$ & 0.006 \\
& Present & 92 & $8[7-10]$ & 0.032 \\
Osmophobia & Present & 127 & $8[7-10]$ & \\
& Absent & 111 & $8[7-9]$ & \\
\hline
\end{tabular}

This could be important for two reasons: first the absence of accompanying symptoms could lead to underdiagnosis of CM. Second, this observation could point to cumulative effects of frequent headaches. CM is described as an altered entity, and there is growing evidence of chronicityrelated structural effects in pain pathways [20]. Interestingly, in our population, headache frequency did not change with age or gender. Duration of illness and intensity scores (VAS) were also similar for both genders. However, headache duration was significantly longer in females. Slater et al. reported longer headache duration

Table 6 Duration of illness (months) according to accompanying symptoms

\begin{tabular}{|c|c|c|c|c|}
\hline $\begin{array}{l}\text { Accompanying } \\
\text { symptoms }\end{array}$ & & $\begin{array}{l}\text { Number of } \\
\text { subjects }(n)\end{array}$ & $\begin{array}{l}\text { Duration of illness } \\
\text { (months) }\end{array}$ & $P$ value \\
\hline \multirow[t]{2}{*}{ Nausea } & Present & 612 & 60 [24-156] & $<0.001$ \\
\hline & Absent & 177 & 36 [10-114] & \\
\hline \multirow[t]{2}{*}{ Vomiting } & Present & 302 & 108 [36-180] & $<0.001$ \\
\hline & Absent & 436 & 36 [12-120] & \\
\hline \multirow[t]{2}{*}{ Phonophobia } & Present & 635 & 60 [24-156] & $<0.001$ \\
\hline & Absent & 157 & 36 [9-96] & \\
\hline \multirow[t]{2}{*}{ Photophobia } & Present & 560 & 72 [24-166] & $<0.001$ \\
\hline & Absent & 227 & 36 [12-96] & \\
\hline \multirow[t]{2}{*}{ Dizziness } & Present & 116 & 114 [36-240] & $<0.001$ \\
\hline & Absent & 545 & 48 [12-120] & \\
\hline \multirow[t]{2}{*}{ Vertigo } & Present & 92 & 82 [24-180] & 0.015 \\
\hline & Absent & 549 & 48 [12-120] & \\
\hline \multirow[t]{2}{*}{ Osmophobia } & Present & 127 & 120 [48-240] & $<0.001$ \\
\hline & Absent & 111 & 48 [12-120] & \\
\hline
\end{tabular}


Table 7 Associations of accompanying symptoms with each other

\begin{tabular}{|c|c|c|c|c|c|c|c|c|}
\hline & & Nausea & Vomiting & Phonophobia & Photophobia & Dizziness & Vertigo & Osmophobia \\
\hline \multirow[t]{3}{*}{ Nausea } & + & & 51.2 & 86.1 & 77.4 & 19.7 & 14.9 & 57.8 \\
\hline & - & & 7.9 & 58.0 & 47.4 & 10.6 & 12.4 & 35.4 \\
\hline & $p$ & & 0.001 & 0.001 & 0.001 & 0.008 & 0.445 & 0.006 \\
\hline \multirow[t]{3}{*}{ Vomiting } & + & 95.3 & & 88.2 & 83.1 & 25.0 & 16.5 & 59.8 \\
\hline & - & 62.6 & & 71.9 & 59.4 & 12.6 & 12.4 & 43.1 \\
\hline & $p$ & 0.001 & & 0.001 & 0.001 & 0.001 & 0.142 & 0.013 \\
\hline \multirow[t]{3}{*}{ Phonophobia } & + & 83.5 & 45.5 & & 82.1 & 20.4 & 16.8 & 59.6 \\
\hline & - & 52.9 & 22.3 & & 27.4 & 6.6 & 6.6 & 19.5 \\
\hline & $p$ & 0.001 & 0.001 & & 0.001 & 0.001 & 0.002 & 0.001 \\
\hline \multirow[t]{3}{*}{ Photophobia } & + & 84.7 & 48.8 & 92.3 & & 21.2 & 16.0 & 59.7 \\
\hline & - & 59.3 & 22.1 & 49.6 & & 9.3 & 10.5 & 28.0 \\
\hline & $p$ & 0.001 & 0.001 & 0.001 & & 0.001 & 0.063 & 0.001 \\
\hline \multirow[t]{3}{*}{ Dizziness } & + & 85.2 & 55.3 & 91.2 & 85.2 & & 50.0 & 71.3 \\
\hline & - & 73.5 & 34.7 & 73.9 & 64.0 & & 6.2 & 32.0 \\
\hline & $p$ & 0.008 & 0.001 & 0.001 & 0.001 & & 0.001 & 0.001 \\
\hline \multirow[t]{3}{*}{ Vertigo } & + & 78.0 & 44.9 & 89.1 & 75.8 & 59.3 & & 65.7 \\
\hline & - & 74.3 & 36.8 & 74.3 & 65.9 & 8.8 & & 39.9 \\
\hline & $p$ & 0.445 & 0.142 & 0.002 & 0.063 & 0.001 & & 0.001 \\
\hline \multirow[t]{3}{*}{ Osmophobia } & + & 86.4 & 53.5 & 93.5 & 88.8 & 60.8 & 43.6 & \\
\hline & - & 71.8 & 36.9 & 70.3 & 67.6 & 22.7 & 21.1 & \\
\hline & $p$ & 0.006 & 0.013 & 0.001 & 0.001 & 0.001 & 0.001 & \\
\hline
\end{tabular}

In this table, + lines indicate subjects who had related symptoms, and - lines indicate subjects who did not have related symptoms

in females in childhood migraine [21]. Adult population studies also report longer headache duration in females [22, 23], suggesting a female gender tendency in $\mathrm{CM}$.

Both duration of illness (months) and headache intensity (VAS) scores were found to be significantly associated with the presence of all accompanying symptoms, including nausea, vomiting, phonophobia, photophobia, dizziness, vertigo, and osmophobia. Although our study covered only CM patients, our results indicate that duration of illness and headache intensity are closely related with accompanying symptoms. Kelman et al. reported that headache intensity was correlated with nausea, vomiting, photophobia, phonophobia, and dizziness, and headache duration was correlated with osmophobia and taste abnormalities [23]. In our results, accompanying symptoms were present as hallmarks of duration of illness and attack intensity in the CM population.

Attack duration was found to be related to the presence of vomiting, phonophobia, and photophobia. However, the presence of other accompanying symptoms-including nausea, osmophobia, vertigo, and dizziness-did not change with varying attack duration. Conversely, Kelman and Tanis reported a correlation between attack duration and osmophobia and taste abnormalities. However, their study also covered EM patients [23]. This difference could point to the effects of chronification on attack phenotype.

Finally, we analyzed the relationships of associated symptoms with each other. The results revealed that nausea, vomiting, phonophobia, and photophobia are closely correlated with each other. Osmophobia (53.4 \%) was also a common accompanying symptom. In addition, the presence of osmophobia was significantly associated with other diagnostic accompanying symptoms. Because ICHD-3 beta did not include other common symptoms accompanying diagnostic criteria, there is insufficient data investigating the predictive value of such symptoms. The diagnostic utility of osmophobia was proposed previously by some authors, especially for Asian migraineurs, in whom photophobia is less common [16]. Similarly, SilvaNeto et al. reported that osmophobia may be a specific marker for migraine and suggested its inclusion within the diagnostic criteria [24]. According to a recent study, the prevalence of both allodynia and osmophobia were higher in chronic than in episodic migraineurs [17]. Same study also reported a relationship between duration of headache and osmophobia.

This study has some limitations. First, the retrospective study design limited our data to monitoring blood pressure, body mass index, and educational-socioeconomic 
status. Second, this study did not include EM patients, so we could not compare our findings with those of EM sufferers. Due to the retrospective design of this study some missing values of the parameters was one of our limitations. But even so power analysis of the data is mostly estimated $80-99 \%$ for variables. However, this study gives us clues about the determinants of phenotype in CM populations.

\section{Conclusion}

In conclusion, our study supports and expands the literature and could be summarized as follows: (1) CM is defined as 15-30 headache days per month, and in our study population, higher numbers of headache days were found to be correlated with less prominent nausea, vomiting, photophobia, and phonophobia; (2) long duration of headache (months) and higher attack intensity (VAS) were found to be correlated with more frequent incidences of all accompanying symptoms investigated; (3) longer attack duration (hours) was found to be correlated with the presence of vomiting and phono-photophobia; (4) duration of headache and attack intensity are the most relevant determinants of CM attack phenotype; (5) osmophobia is also common in CM and is associated with diagnostic accompanying symptoms; and (6) dizziness and vertigo were less frequent in the $\mathrm{CM}$ population and do not have a prominent relationship with other diagnostic accompanying symptoms.

Future studies focusing on clinical presentation and accompanying symptoms could provide additional data to shed light on the phenotypes, classifications, and pathophysiological pathways of chronicity.

\section{Abbreviations \\ CM: chronic migraine; ICHD-3 beta: the third edition of the International Classification of Headache Disorders; VAS: visual analogue scale.}

\section{Competing interests}

The authors declare that they have no competing interests.

\section{Authors' contributions \\ OÖY, DU, AÖ, MS, and MAS participated in the alignment and drafted the manuscript. OÖY, DUI, AÖ, MAS, AS participated in the design of the study and performed the statistical analysis. DU, AO conceived of the study, and participated in its design and coordination and helped to draft the manuscript. All authors read and approved the final manuscript.}

\section{Acknowledgements}

This study is conducted as a part of Turkish Headache Database Project. Turkish Headache Database Project is supported by Turkish Neurological Society and unsolicited grant from Allergan INC.

\footnotetext{
Author details

${ }^{1}$ Neurology Department, Istanbul Education and Research Hospital, Kasap Illyas Mah. Org. Abdurrahman, Nafiz Gürman Cd, PK: 34098 Fatih, Istanbul, Turkey. ${ }^{2}$ Neurology Department, Istanbul University Cerrahpasa School of Medicine, İstanbul, Turkey. ${ }^{3}$ Neurology Department, Mersin University School of Medicine, Mersin, Turkey. ${ }^{4}$ Biostatistics Department, Düzce University School of Medicine, Düzce, Turkey. ${ }^{5}$ Neurology Department, Kocaeli University School of Medicine, Kocaeli, Turkey.
}

Received: 29 January 2016 Accepted: 8 March 2016

Published online: 15 March 2016

\section{References}

1. Headache Classification Committee of the International Headache Society (IHS) (2013) The International classification of headache disorders, 3rd ed. (beta version). Cephalalgia 33(9):629-808

2. Natoli JL, Manack A, Dean B, Turkel CC, Stovner L, Lipton RB (2009) Global prevalence of chronic migraine: a systematic review. Cephalalgia 31:1116-1130

3. Bigal ME, Serrano D, Buse D, Scher A, Stewart WF, Lipton RB (2008) Acute migraine medications and evolution from episodic to chronic migraine: a longitudinal population-based study. Headache 48(8):1157-1168

4. Buse DC, Manack AN, Fanning KM, Serrano D, Reed ML, Turkel CC, Lipton RB (2012) Chronic migraine prevalence, disability, and sociodemographic factors: results from the American Migraine Prevalence and Prevention Study. Headache 52(10):1456-1470

5. Ertas M, Baykan B, Orhan EK, Zarifoglu M, Karli N, Saip S, Siva A (2012) Oneyear prevalence and the impact of migraine and tension-type headache in Turkey: a nationwide home-based study in adults. Headache 13(2):147-157

6. Lipton RB, Silberstein SD (2015) Episodic and chronic migraine headache: breaking down barriers to optimal treatment and prevention. Headache 55(S2):103-122

7. Sanderson JC, Devine EB, Lipton RB, Bloudek LM, Varon SF, Blumenfeld AM, Sullivan SD (2013) Headache-related health resource utilisation in chronic and episodic migraine across six countries. J Neurol Neurosurg Psychiatry 84:1309-1317

8. Buse DC, Manack A, Serrano D, Turkel C, Lipton RB (2010) Sociodemographic and comorbidity profiles of chronic migraine and episodic migraine sufferers. J Neurol Neurosurg Psychiatry 81:428-432

9. Lipton RB (2011) Chronic migraine, classification, differential diagnosis, and epidemiology. Headache 51(S2):77-78

10. Bigal ME, Lipton RB (2006) Modifiable risk factors for migraine progression. Headache 46(9):1334-1343

11. Schwedt TJ (2014) Chronic migraine. BMJ 348:g1416

12. Aurora SK, Barrodale PM, Tipton RL, Khodavirdi A (2007) Brainstem dysfunction in chronic migraine as evidenced by neurophysiological and positron emission tomography studies. Headache 47(7):996-1003

13. Noseda R, Burstein R (2013) Migraine pathophysiology: anatomy of the trigeminovascular pathway and associated neurological symptoms, cortical spreading depression, sensitization, and modulation of pain. Pain. 154:44-53.

14. Welch KMA, Nagesh V, Aurora SK, Gelman N (2001) Periaqueductal gray matter dysfunction in migraine: cause or the burden of illness? Headache 41(7):629-637

15. Aurora SK (2009) Spectrum of illness: understanding biological patterns and relationships in chronic migraine. Neurology 72(5):8-13

16. Wang YF, Fuh JL, Chen SP, Wu JC, Wang SJ (2012) Clinical correlates and diagnostic utility of osmophobia in migraine. Cephalalgia 32(16):1180-1188

17. Lovati C, Giani L, Castoldi D, D'Alessandro CM, DeAngeli F, Capiluppi E, Mariani C (2015) Osmophobia in allodynic migraineurs: cause or consequence of central sensitization? J Neurol Sci 36(1):145-147

18. Olesen J, Steiner TJ (2004) The International classification of headache disorders, 2nd edn (ICDH-II). J Neurol Neurosurg Psychiatry 75(6):808-811

19. Kelman $L$ (2006) Pain characteristics of acute migraine attack. Headache 46(6):942-953

20. Bernstein C, Burstein R (2012) Sensitization of the trigeminovascular pathway: perspective and implications to migraine pathophysiology. J Clin Neurol 8:89-99

21. Slater S, Crawford MJ, Kabbouche MA, LeCates SL, Cherney S, Vaughan P, Hershey AD (2009) Effects of gender and age on paediatric headache. Cephalalgia 29(9):969-973

22. Bolay H, Ozge A, Saginc P, Orekici G, Uludüz D, Yalın O, Siva A, Bıçakçi \$, Karakurum B, Öztürk M (2015) Gender influences headache characteristics with increasing age in migraine patients. Cephalalgia 35(9):792-800

23. Kelman L, Tanis D (2006) The relationship between migraine pain and other associated symptoms. Cephalalgia 26(5):548-553

24. Silva-Néto RB, Peres MFP, Valença MM (2014) Accuracy of osmophobia in the differential diagnosis between migraine and tension-type headache. J Clin Neurol 339:118-122 